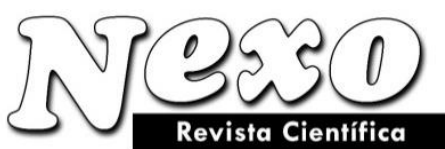

Vol. 34, No. 01, pp. 390-403/Marzo 2021

\title{
The influence of formation water on mass transfer in the coal seam
}

\section{Influencia de formaciones de agua en la transferencia de masa en vetas de carbón}

\author{
Vitaly A. Trofimov \\ Institute of Comprehensive Exploitation of Mineral Resources Russian Academy of Sciences. \\ asas_2001@mail.ru \\ (recibido/received: 17-November-2020; aceptado/accepted: 05-January-2021)
}

\begin{abstract}
The construction of a model of mass transfer in coal seams involves consideration of a number of conceptual provisions related to various aspects of this process. First, the theoretical aspects of the phenomena responsible for mass transfer must be considered. Namely, the nature of the fluid flow in a fracturing porous medium is concretized, the role of the stress state of the medium is determined, and the thermodynamic component of the processes is estimated. Secondly, the initial conditions of the state of systems must be determined, which exist in the array before the technogenic interference into it, i.e. before the start of formation development. Thirdly, the corresponding equations must be written out describing the ongoing processes, which actually form the basis of the model. Fourth, it is necessary to develop or adapt methods for solving the corresponding equations in conjunction with the initial and boundary conditions. The obtained solutions should be tested on known analytical solutions to confirm the performance of compiled algorithms for their accuracy and convergence.

Keywords: Coalbed methane; Formation water; Gas and water pressure, Saturation; Permeability; Twophase filtration.
\end{abstract}

\section{RESUMEN}

La construcción de un modelo de la transferencia de masa en capas hulleras requiere considerar unas provisiones conceptuales acerca de los varios aspectos de este proceso. Primero, hay que considerar los aspectos teóricos del fenómeno responsables por las transferencia. Es decir se concretiza la naturaleza del movimiento de fluidos en un medio agrietado esponjoso, determina el papel del estado de tensión del medio y avalora el componente termodinámico de los procesos. En segundo lugar, hay que determinar las condiciones iniciales del estado del sistema que existen en el macizo antes de la interferencia tecnogénica en este, o sea, antes de trabajar la capa. En tercer lugar, hay que componer las ecuaciones correspondientes para describir los procesos en curso que forman la base del modelo en realidad. En cuarto lugar, es necesario desarrollar o adaptar métodos para resolver las ecuaciones correspondientes junto con las condiciones iniciales y límites. Hay que probar las soluciones derivadas en soluciones analíticas ya conocidas para confirmar la funcionalidad de los algoritmos desarrollados en términos de su convergencia y precisión.

Palabras claves: Metano de las capas; Agua intersticial, Presión de gas y agua; Saturación, Permeabilidad, Filtración bifásica 


\section{INTRODUCTION}

Traditionally, models of filtration in a porous medium are based on the principles of spatial and temporal compatibility of at least two processes: fluid transfer in a certain continuous medium, which is described by the corresponding differential equations, and the deformation process of the same medium, also considered as a continuous one with the corresponding equations describing the change stress-strain state of the medium. In addition, it is believed that for a coal seam, a source of methane is located at each of its points, which can be released into the filtration flow or it is absorbed. Thus, with this approach, the filter medium does not contain pores and cracks, which are explicitly taken into account. Their influence is determined by the porosity and permeability parameters included in the filtration equation and the deformation-strength parameters of the deformation model.

To date, numerous attempts have been made to calculate the permeability, based on various ideas about the structure of coal fracturing, starting from the presentation of the fractured pore space of coal as a system of capillaries, or a set of spherical particles (Akkutlu et al., 2016).

A further development of the theory of filtration in fractured porous media was the concept of a dual porosity and multicontinuum model (Aziz \& Settari, 2002; Barenblat et al., 1960, 1972). Fracture systems are the defining elements in the formation of the permeability of the coal seam, forming a single filtration space. At the same time, rock matrices separated by fractures contain a large number of pores, along which fluids are also transferred. The interaction of such structures in the models is specified by introducing the flow functions specified at the boundary between the rock matrix and fractures, and the determination of which is the key problem of multicontinuum models (Bourgeat, 1984; Brezzi \& Fortin, 1991; Douglas et al., 1993).

This model was developed in the works of Barenblatt (Aziz \& Settari, 2002), Warren and Root (Efendiev et al., 2015). Then Kazemi (Ewing , 2001) expanded the Warren-Root model for a two-phase fluid. Later, Douglas and Arbogast (Barenblatt et al., 1984) used the averaging procedure in single-phase and multiphase filtration models for double porosity.

There is also an approach associated with the introduction of two complete sets of variables at each point, corresponding to mass transfer in a porous medium, as well as along cracks (Glushkova \& Papin, 2019). At the same time, there is a relationship between these flows, in the form of an overflow, which is considered proportional to the pressure difference in pores and cracks. In this case, mass transfer is still described within the framework of the continuum approach.

In the case of sufficiently large unconnected fractures, models for direct accounting of fracture flow were built, such as a Discrete Fracture Model (DFM) or an Embedded Fracture Model (EFM). The discrete fracture model allows one to take into account the feature associated with the fracture structure at the grid level and set the corresponding properties on it (Gong et al., 2008; Kalinkin \& Laevsky, 2015).

This approach requires large computational resources, since it is really necessary to take into account a large number of cracks. Such consideration of cracks in the approximation of the problem leads to a large number of unknowns.

It should be noted that some cracks whose dimensions are small compared to the reservoir size cannot be individually considered in the design scheme. In contrast to the DFM method, the EFM method allows averaging many small cracks and reducing the dimension of the system to be solved (Kazemi et al., 1976; Konovalov, 1994). 
In addition to methane, the coal seam contains a significant amount of water, which is approximately equal to the weight of methane. If there are wells drilled through the reservoir, both methane and formation water are released from them. Water, methane and sorbing coal interact with each other quite difficult during technogenic impact on the reservoir. At sufficiently high pressures in the pore space, water can displace methane from pores and cracks, partially into the free space of workings and wells, as well as into the sorbed state in coal and completely fill the filtration space. After that, pure water without methane will be released into the well (Kuznetsov \& Trofimov, 1998). Thus, in the coal seam, there is a two-phase filtration of immiscible and, we can assume, mutually insoluble fluids - water and methane.

An extremely large number of works have been devoted to the theoretical description of two-phase filtration. It should be noted that, based on the needs of practice, most of them are focused on the problems of the oil industry, where the competing fluids are water and oil moving in a neutral permeable medium (sandstone).

One of the existing approaches for numerical simulation of filtration processes in fractured-porous media of a two-phase incompressible fluid is described in (Glushkova \& Papin, 2019; Kuznetsov \& Trofimov, 1998, 1999, 2000; Kuznetsov \& Krigman, 1978). The mathematical model formulated in terms of "velocity-pressure-saturation" (Kuznetsov \& Trofimov, 2012) was used in the works of Yuvinga R. et al. (Kuznetsov \& Trofimov, 2008).

One more class of two-phase filtration problems should be mentioned. This is the movement of two immiscible liquids, each of which occupies a certain area of the filtration space. In this case, we are talking about the stability of the border of their contact. In this case, the piston effect takes place when the fluids mutually displace each other (Kuznetsov \& Trofimov, 2005).

The following circumstance that allows us to consider the approach we are developing as new and unique should be noted. First, in our case we are talking about a coal seam, which is inherent in the ability to sorb methane. When constructing the model, it can be assumed that at each point of the considered area there is a source/sink of methane, which can be added or decreased from the flow as appropriate. Secondly, water is also present in almost every pore and crack of coal matter, being a constituent part of the coal seam, apparently formed in the process of coal metamorphism. That is, each crack-pore contains both gas and water. And they migrate through the same filtration channels. At the same time, when building a filtration model, it is natural to assume that a certain "effective" mixture with averaged parameters is being filtered. There is no such situation in oil filtration and in all considered models.

\section{MATERIALS AND METHODS}

\subsection{Mathematical model of mass transfer in a coal seam}

Taking into account these features of coal seams, the author considers the formulation and solution of the problem of joint filtration of water and gas in a coal seam. Let us write down the general relations underlying the description of various gas-dynamic processes occurring in coal substance. This includes both quasi-static transfer and outflow processes, and dynamic types of sudden coal and gas outbursts. In this case, a lot of different problems arise, the solution of which, with all their diversity, has a common methodological basis.

For simplicity, the author will consider the problems in a one-dimensional formulation, which allows covering a wide range of practical problems. In fact, these are all tasks associated with the inflow of fluids to an extended working, whether it is a lava face, cut openings or drifts. 
Important tasks are related to the assessment of the flow of methane and water from the roof rocks into the developed space. In all these cases, the one-dimensional flow is implemented in a significant part of the massif and constitutes the main part of the flow, with the exception of rather small areas, mainly at the junctions of the workings.

The equations of continuity and motion of the mixture in the filtration flow, as well as the continuity of water, will be written in the form (Nigmatulin, 1987; Trofimov \& Filippov, 2015; Trofimov, 2014; Vabishchevich \& Grigoriev, 2016).

$$
\begin{gathered}
\frac{\partial \rho u}{\partial x}+\frac{\partial Q}{\partial t}=0, \quad u=-\frac{k}{\mu} \frac{\partial p}{\partial x}, \\
\frac{\partial \rho_{w} u_{w}}{\partial x}+\frac{\partial s m \rho_{w}}{\partial t}=0
\end{gathered}
$$

Where

$$
Q=m \rho+\frac{a b p}{1+b p},
$$

$\rho, u, \mu$ - density, filtration rate and viscosity of the mixture, $k, m$ - permeability and porosity, $s$ - water saturation, i.e. the proportion of porosity occupied by water, $\rho_{w}, u_{w}$ - density and filtration rate of water, $p$ - gas pressure, $a, b$ - sorption constants.

At the gas constant $R$ and absolute temperature $T$, the pressure is related to the gas density $\rho_{g}$ by the equation of state

$$
p=\rho_{g} R T
$$

In the accepted designations, $m_{s} \rho_{w}-$ the amount of water per unit volume of coal, and $m(1-s) \rho_{g}-$ the amount of free gas. Therefore, to determine the density of the mixture, we have:

$$
\rho=s \rho_{w}+(1-s) \rho_{g}
$$

Obviously, $\rho_{w}$ can be considered constant in comparison with $\rho_{g}$.

The amount of jointly flowing water and gas, or just a mixture, through a unit area of a porous medium per unit of time is equal to $\rho_{u}$. In this case, the amount of water flows $-\rho_{w} u_{w}$, and the amount of gas $-\rho_{g} u_{g}$. Therefore

$$
\rho u=\rho_{w} u_{w}+\rho_{g} u_{g}
$$

As a result, for a single-speed flow, we have

$$
u w=s u, \quad u_{g}=(1-s) u
$$

It should be noted that when determining the filtration rate, the movement of the mixture should be understood as the joint movement of water and gas with a certain uniform viscosity. Regarding the viscosity of such a mixture, it is possible to say with complete certainty only that it depends on water saturation and it is related to the viscosities of water itself $\mu_{w}$ and gas $\mu_{g}$.

In this work, to determine it, the author uses the empirical Kozeny formulas, which describe the phase permeability of water $k_{w}$ and gas $k_{g}$ depending on water saturation (Kuznetsov \& Trofimov, 1998). 


$$
\frac{k_{w}}{k}=s^{4}, \quad \frac{k_{g}}{k}=(1-s)^{3}(1+3 s)
$$

According to (7), with a general pressure gradient in the mixture of water and gas, we have

$$
\frac{k}{\mu}=\frac{k_{w}}{\mu_{w}}+\frac{k_{g}}{\mu_{g}}
$$

Replacing here $k_{w}, k_{g}$ by their expressions in (8), after simple transformations we obtain

$$
\frac{\mu}{\mu_{w}}=\frac{1}{s^{4}+\frac{\mu_{w}}{\mu_{g}}(1-s)^{3}(1+3 s)}
$$

Regarding the permeability $k$, the author notes that in specific reservoir conditions it depends mainly on the compressive stresses in the reservoir and the amount of gas sorbed by coal. In this case, the amount of sorbed gas is indirectly related to the swelling and shrinkage of coal, i.e. with the corresponding reservoir deformations and stresses arising from the sorption/desorption of methane. Ultimately, both the permeability and the viscosity of the mixture, in addition to the aforementioned parameters, depend on saturation due to (8)-(10), i.e. the proportion of water in the flow.

\subsection{Formulation and solution of the problem of mixture movement in the edge of the reservoir}

Let us further consider the filtration problem related to the movement of gas and water in the zone of sudden extraction of the edge of the formation.

It is known that under certain conditions there is a sudden squeezing out of the edge of the formation (Vasilyeva et al., 2016, 2017, 2018), accompanied by unloading and loosening of a certain zone in the edge of the formation. At the same time, it can be assumed that a coal massif with constant pressure, permeability, porosity, saturation, and moisture is formed in such a zone. When a sudden extraction occurs within this zone, the elastic deformation energy of coal destroys it, the seam in it becomes permeable and thus the beginning of the filtration gas flow towards the bottom is initiated. At the same time, the formation remains in its natural state outside the extraction zone, i.e. impenetrable (Warren \& Root, 1963). At the same time, this transformation of the edge part of the formation occurs in a dynamic mode with a characteristic time of $\sim 1$ s, i.e. from the point of view of subsequent filtration processes - instantly.

Thus, the author considers the filtration problem for a bounded region in which at the initial moment of time the initial pressure is $\mathrm{p}=50 \mathrm{~atm}$ and, accordingly, the amount of sorbed gas, water saturation $-\mathrm{s}=0.5$, constant permeability $-\mathrm{k}=10-2 \mathrm{mD}$ and porosity $-\mathrm{m} 0=0.03$. At the boundary at $\mathrm{x}=0$, the free exit of the mixture into the open space is specified, i.e. $\mathrm{p}=1$ atm. As for the right boundary $\mathrm{x}=\mathrm{L}=20 \mathrm{~m}$, located in the depth of the formation, it is considered impenetrable, i.e. there is no inflow of the mixture from the outside. In addition, it is believed that at the moment of the sudden extraction, the advance of the bottomhole has stopped and no further change in the size of the extraction zone occurs. The situation when the face moves at a constant speed was considered earlier (Zakharov et al., 2016) in the framework of the 
analytical approach. In this case, a water and gas filtration zone is formed in front of the face, the dimensions of which become unchanged over time in relation to the moving front.

\section{RESULTS AND DISCUSSION}

\subsection{Mass transfer of water and methane in the edge of the reservoir}

At present, the finite difference method is a rather effective approach to solving such problems with a nonlinear parabolic equation. At the same time, the use of implicit difference schemes allows relatively free operation of steps in time and space.

Figure 1 shows the profiles of the pressure distribution $\mathrm{p}$ along the length of the calculating area for the period $0<\mathrm{t}<100$ days with an interval of 1 day. During this time, the pressure on the right border dropped from $50 \mathrm{~atm}$ to $\sim 25 \mathrm{~atm}$, i.e. it formed a jump of $25 \mathrm{~atm}$.

From a technological point of view, consideration of the development of gas removal from the extraction zone within 100 days seems to be of only theoretical interest, but in practice it can be only a few days, when the filtration has not yet reached the right end, and there is practically no crossflow over this boundary.

Or, with a large induced permeability in the squeezing zone, when the filtration still reaches the right boundary, a constant pressure can be put on it, considering it as a power supply circuit.

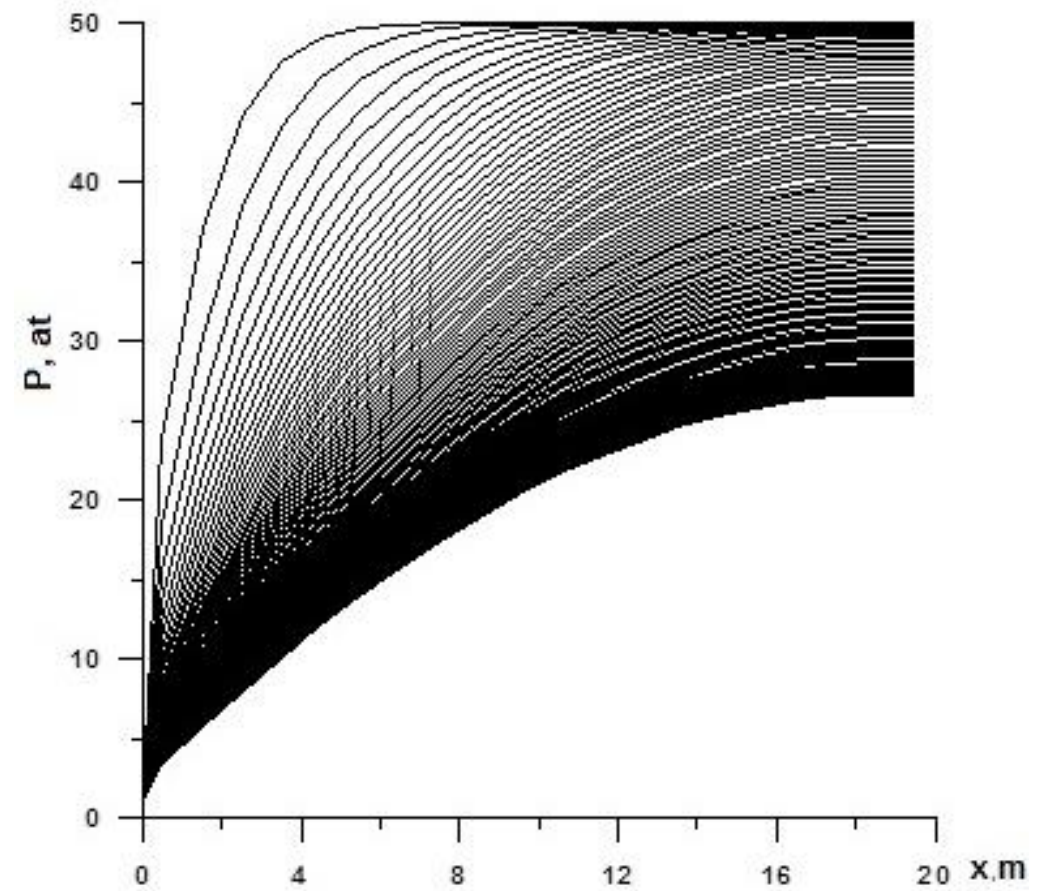

Figure 1. Distribution of gas and water pressure in a coal seam at different times with an interval of 1 day

Complicating the problem, we can assume that there is a certain inflow of water and gas at the right border, which is associated with the pressure gradient that occurs on it over time. In the presented example, this does not seem to be fundamental, since the principal possibility of modeling two-phase filtration by a mixture flow is considered. 


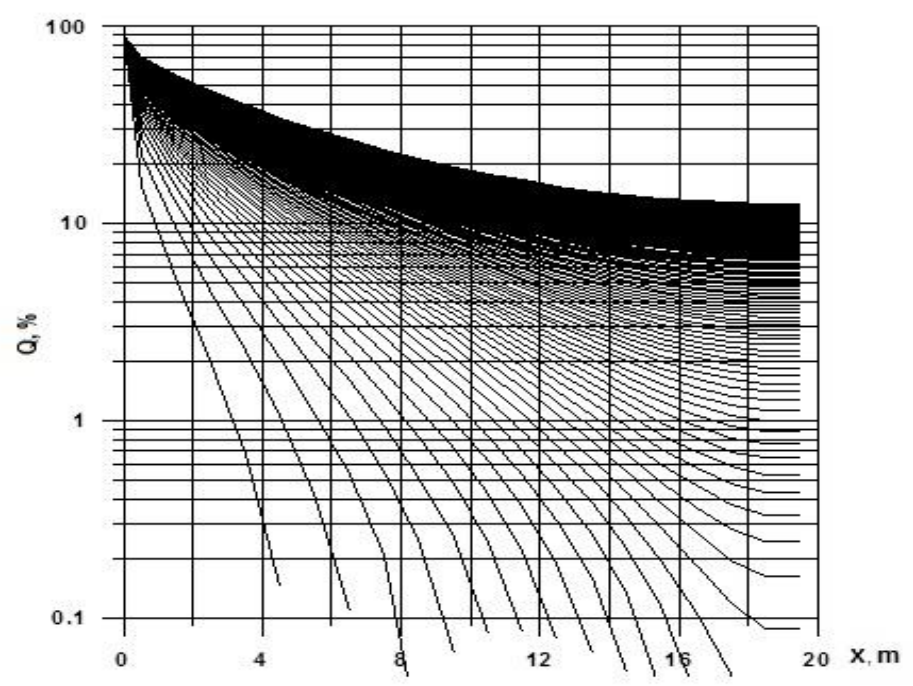

Figure 2. Distribution of the amount of sorbed gas as a percentage of the initial content in the coal seam at different points in time with an interval of 1 day

Figure 2 shows the kinetics of gas desorption from the area over the same time period. The curves show the percentage of gas desorbed from coal. Near the free surface, it is almost $100 \%$, while near the other boundary it is slightly more than $10 \%$.

All the graphs presented have limiting curves, to which all the obtained curves are condensed for different time values $\mathrm{t}$. We can assume that for $\mathrm{t}>\mathrm{tk}$ all of them actually coincide. In the case under consideration: tk $~ 60$ days.

In the area under consideration, at the initial moment of time, $300 \mathrm{~L}$ of water was contained in the cracks, of which $\sim 80$ liters came out through the free surface after 100 days. The dynamics of this process is shown in Figure 3.

At the same time, the amount of sorbed gas was initially $416 \mathrm{~kg}$, which produced $\sim 130 \mathrm{~kg}$, or $190 \mathrm{~m} 3$ for normal conditions. That is, on average, about $2 \mathrm{~m} 3$ of gas from each square meter of free surface per day.

The change in water saturation over time along the length of the area (as shown in Figure 4) is of interest. The initial water saturation of 0.5 rather quickly (over the first day) falls to $\sim 0.39$ at the boundary $x=0$, and then slowly decreases to $\sim 0.325$ on the hundredth day. This means that the initial moisture content in the coal seam, which was $\sim 1.1 \%$, falls to $\sim 0.7 \%$, i.e. by $\sim 40 \%$. With greater water saturation, this difference will be even greater, and therefore, the moisture content of the reservoir should be judged from samples taken from the bottom-hole surface with some correction. 


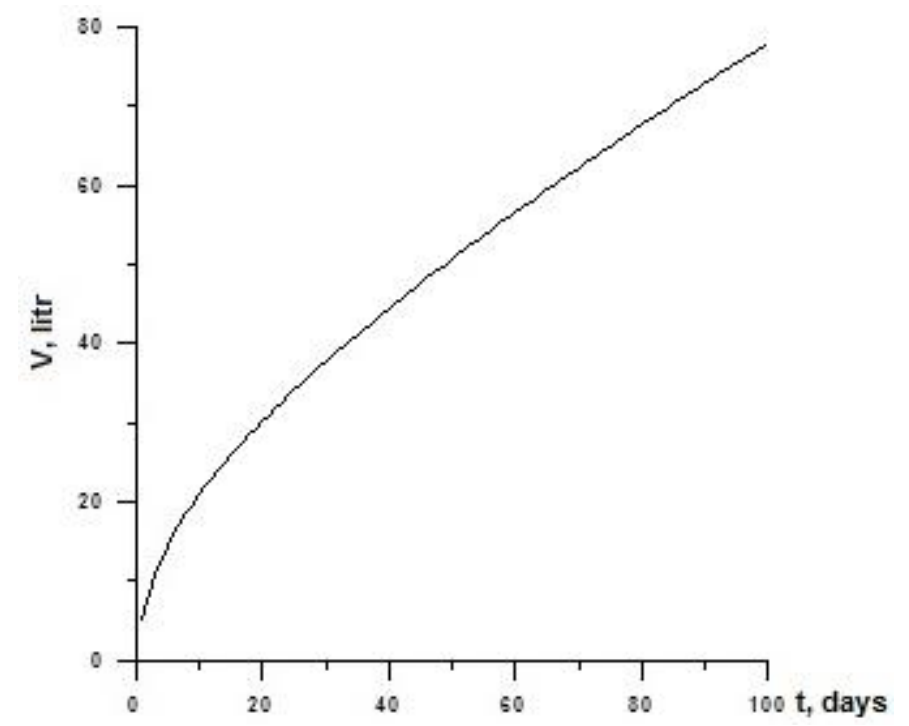

Figure 3. The amount of water released from the reservoir depending on the time

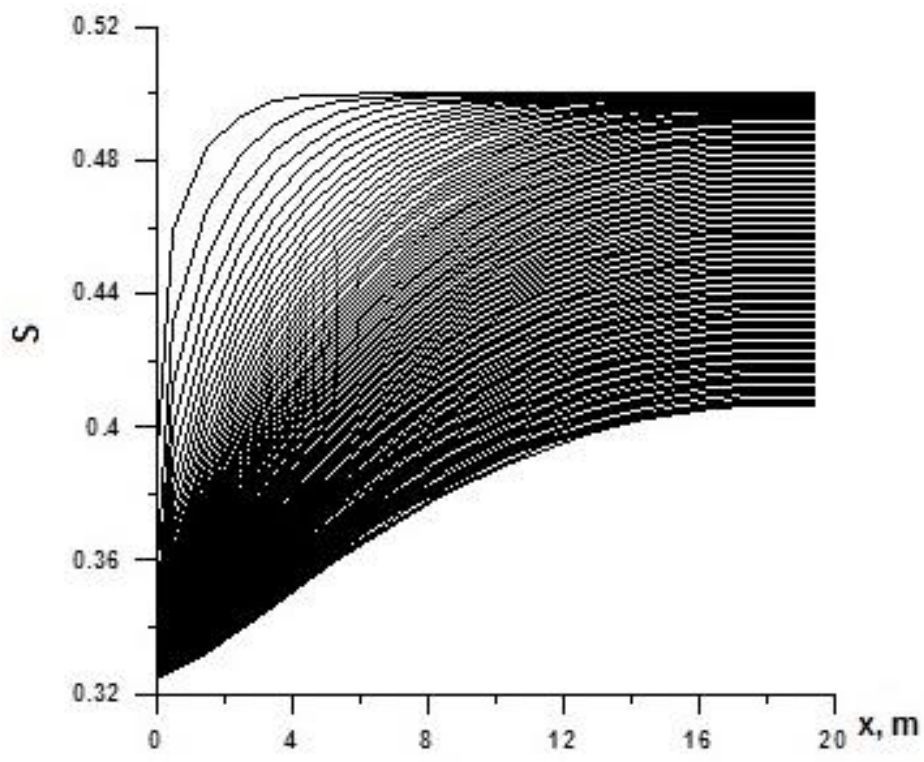

Figure 4. Change in water saturation of the edge of the reservoir in time with an interval of 1 day

\section{2. "Locking" effect of reservoir water on mass transfer in the edge of the formation}

Next, the author considers a similar problem with slightly different parameters for modeling the mass transfer mode of pure water. A filtration problem is considered for a limited area, in which at the initial moment of time the initial pressure is $\mathrm{p}=80 \mathrm{~atm}$ and, accordingly, the amount of sorbed gas, water saturation $-s=1.0$, constant permeability $-k=5 \mathrm{mD}$, and porosity is $m_{0}=0.03$. At the boundary at $x=0$, the free flow of the mixture into the open space is set at $p=25 \mathrm{~atm}$. As for the right boundary $x=L=250 \mathrm{~m}$, located in the depth of the formation, then a constant pressure $(p=80 \mathrm{~atm})$ is set on it, and the mixture flows from the outside. In addition, in the entire region, the amount of sorbed gas $q=20.83 \mathrm{~kg} / \mathrm{m}^{3}$ is 
specified, which corresponds to a sorption pressure of 50 atm with the values of sorption constants $a=25$ $\mathrm{kg} / \mathrm{m}^{3}$ and $b=0.1$.

Figure 5 shows the pressure distribution in the area under consideration at different points in time with an interval of 1 day. The limiting pressure distribution is displayed by a straight line (0.25)-(250-80) to which all distributions gradually approach at intermediate time points. Starting from $t=\sim 50$ days, all of them practically coincide with this straight line. It can be seen from the graph that at distances less than $\sim 150 \mathrm{~m}$ from the right side of the area with a pressure of $p=25 \mathrm{~atm}$, the pressure in the calculated area is less than the sorption one, i.e. desorption and filtration of methane are possible. At $x>\sim 115 \mathrm{~m}$, all the gas is in the sorbed state and its mass transfer is impossible.

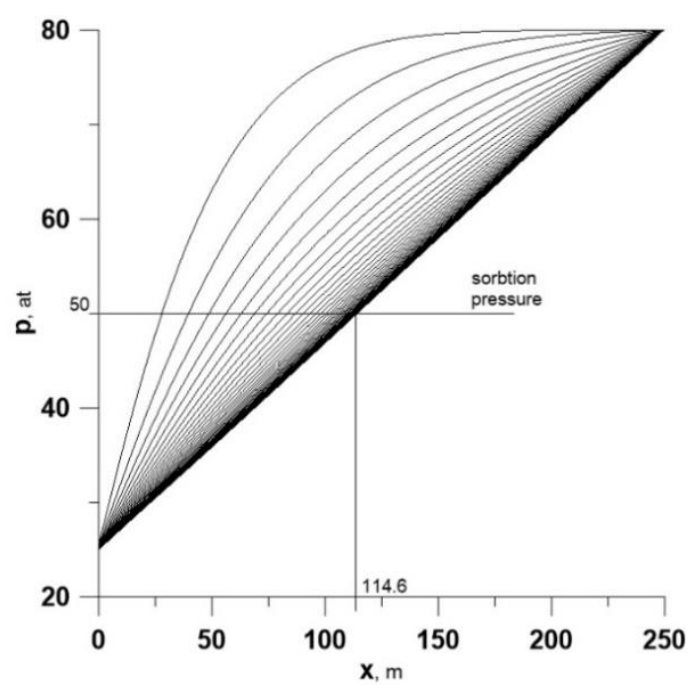

Figure 5. Distribution of gas and water pressure in a coal seam at different points in time with an interval of 1 day at a pressure in the supply circuit above the sorption pressure (80 atm)

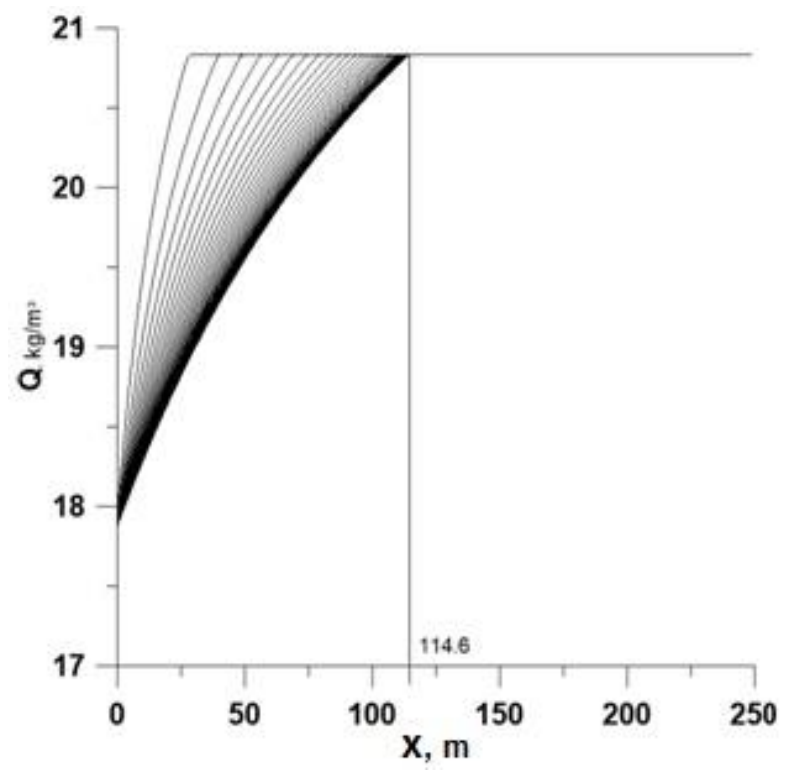


Figure 6. Distribution of the amount of sorbed gas in a coal steam at different points in time with an interval of 1 day at a pressure in the supply circuit above the sorption pressure (80 atm)

Figures 6 and 7 depict this situation in terms of the amount of sorbed gas and reservoir saturation. Figure 6 shows that the sorbed gas came out only from the area in which the "mixture" pressure is less than the sorption pressure $(\sim 115 \mathrm{~m})$. This also applies to the saturation of the reservoir with water, which is given for a period of up to 50 days. On the first day, it quickly fallas to 0.94 , and then begins to increase, including due to the inflow through the right border (not shown in the figure).

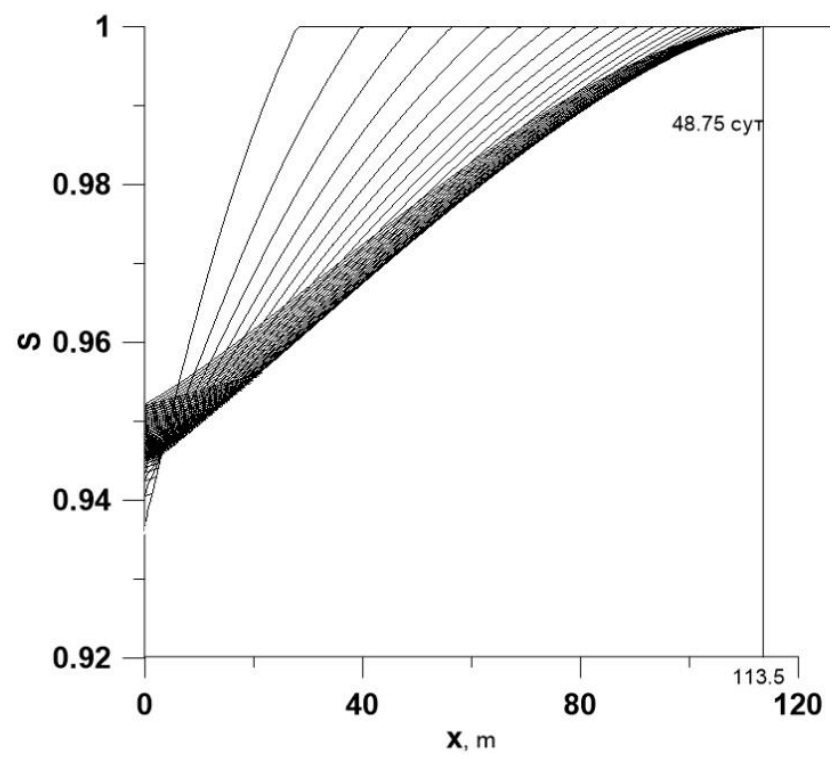

Figure 7. Distribution of saturation of a coal seam with water at different points in time with an interval of 1 day at a pressure in the supply circuit above the sorption pressure (80 atm)

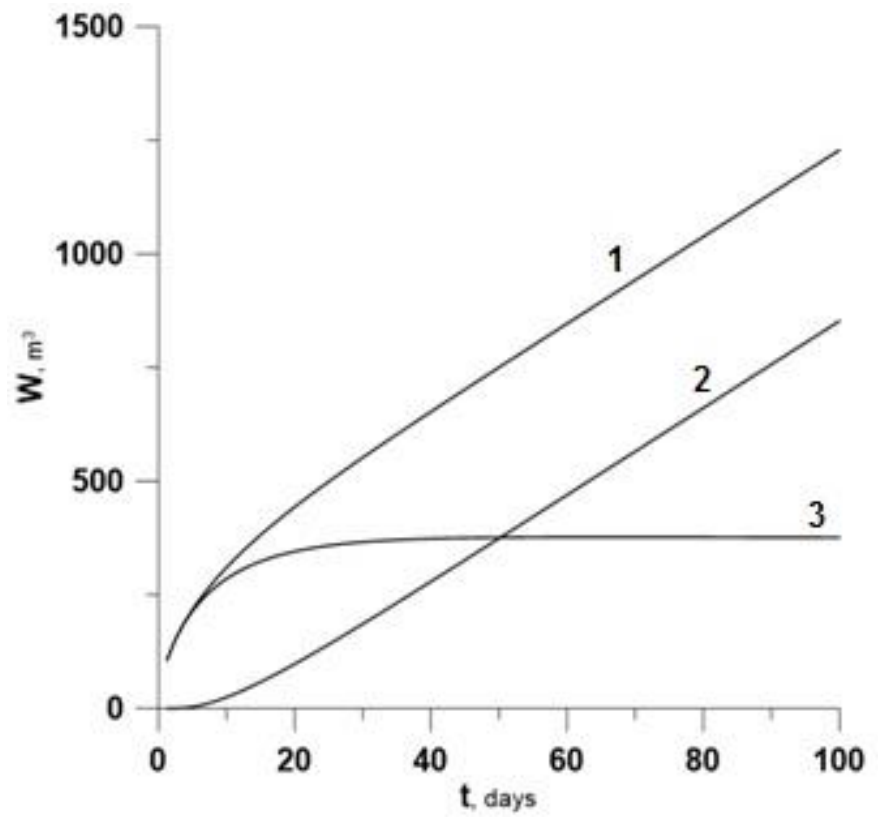

Figure 8. Water content in the edge of the reservoir: 1 - free surface outlets (at $x=0), 2$ - inflow through the feed loop, 3 - amount of water in the reservoir 
The dynamics of water mass transfer is shown in Figure 8, which shows the inflow of water through the right boundary (2) and outflow at the left boundary (1), as well as changes in the amount of water in the computational domain for 100 days. A dynamic change in this amount is observed in the first $\sim 40$ days, after which the filtration mode turns into a stationary mode, when the inflow and outflow of water across the boundaries are compared.

It can be seen from the graph in Figure 6 that the gas output is limited only by a part of the calculated area in which it can be desorbed from coal. It should be noted that the border at $\sim 115 \mathrm{~m}$ does not move after 50 days, because the water pressure at $x>\sim 115 \mathrm{~m}$ locks methane in a sorbed state in coal.

This article discusses the fundamental provisions underlying the model of mass transfer of fluids in a coal seam. This concept of the reservoir as a block structure, in which filtration is carried out through a single "filtration space" formed by a system of cracks and pores, and sorption-desorption (diffusion) occurs in the "sorption space", i.e. in blocks of coal matter.

The concept of the permeability of coal matter and governing laws (Darcy's law) is considered. Equations of gas and water motion in a permeable medium and corresponding initial and boundary conditions for typical problems are written.

A finite-difference algorithm for solving these equations was compiled; it was tested on a number of analytical solutions, which showed its convergence and stability.

It should be noted that the constructed algorithm is quite universal and allows one to describe various situations related to mass transfer in the reservoir. With the appropriate formulation of the problem, i.e. by specifying the initial and boundary conditions, it is possible to describe the gas outflow both from the well and from the bottom-hole surface, from the interbed and adjacent coal seams, with preliminary degassing of the seam from the surface using hydraulic fracturing. The algorithm allows one to describe both quasistationary and dynamic processes, in the form of sudden gas outbursts, as well as coal and gas.

The constructed model of mass transfer in the coal seam allows us to consider in detail the features of interaction of methane and reservoir water in the coal seam under technogenic impact on it. First, the pressure in the gas and water phases in the cracks is the same. In this case, the water pressure is mainly determined by the rock pressure, i.e. compressive stresses acting in the array. They compress the coal, and along with it, the cracks, trying to reduce their lumen, which leads to compression of water and gas in them. The compressibility of water is many orders of magnitude less than that of a gas and, therefore, it (compressibility of water) can be neglected and it can be assumed that the pore compression and the gas compression are equal. In other words, water in the pore simply takes away part of the space from the gas, i.e. it reduces gas porosity.

Since the water pressure can be arbitrary, including high enough, exceeding the sorption pressure for the gas, then, taking into account its sorption equilibrium, a situation may arise when all the gas in the pore passes into the sorbed state into the surrounding coal. In this case, all the pores will be filled with water, which will be filtered through them under the influence of the pressure gradient in the reservoir. In this case, the water saturation $s$ will be equal to 1 .

Subsequently, as water leaves the reservoir on the free surface, its pressure will begin to fall down to sorption pressure. During this period of time, only pure water without gas will be released from the surface. When the pressure in the pores reaches the sorption value, desorption of gas into the pores will begin, followed by exit to the free space (well, production). 
It should be noted that the situation is completely repeated when calculating inflows to the well, for which the algorithm for calculating the filtration (1) should be slightly changed due to the fact that the ratios used to calculate the gradients in the case of a one-dimensional flat flow and a one-dimensional axisymmetric flow are slightly different. The above ratios and graphs for mass transfer for a one-dimensional flat flow and at a pressure at the left boundary of $1 \mathrm{~atm}$, i.e. when the well is completely open, they actually coincide to within a coefficient. The situation changes dramatically if this pressure exceeds the sorption pressure. Calculations within the framework of the above algorithm show the complete absence of gas desorption and migration in the reservoir, and the output at the left boundary into the well of pure water.

The situation when at the left border, i.e. in a well or in the development, at the initial moment, a pressure of 50at is created, seems almost unrealistic. However, if the mixture is released into a closed well, then the pressure in it gradually increases and may exceed the sorption pressure. Then the filtering mode described above will be implemented.

\section{CONCLUSIONS}

The current state of affairs in the field of coal seam degassing seems to be far from optimal. At the same time, due to insufficient knowledge of the processes occurring in this case and a lack of knowledge about the values of parameters that determine them, there is a significant gap in the scientific substantiation of degassing measures. This type of problem is mainly solved by sometimes unreasonable increase in the number of degassing wells, which is undesirable from an economic point of view.

The article presents a model of two-phase filtration of methane and formation water in the edge of the coal seam. The configuration of the problem under study is set using initial and boundary conditions, the variation of which makes it possible to consider a fairly wide range of problems on the mass transfer of fluids to the free wall of a coal seam. In this case, both transport in the final region, setting the conditions on the supply circuit (pressure, inflows of water and gas, etc.), and, by increasing the length of the the calculated area, under certain conditions, transport in unlimited space can be modeled.

The used model approach is more adequate from a physical point of view and reflects the essence of the processes occurring in the edge of the reservoir. Moreover, it is much simpler than existing methods in terms of numerical modeling.

\section{ACKNOWLEDGMENTS}

This work was carried out with the financial support of the Russian Foundation for Basic Research No. 18-05-00936.

\section{REFERENCES}

Akkutlu, I.Y., Efendiev, Y., \& Vasilyeva, M.,V. (2016). Multiscale model reduction for shale gas transport in fractured media. Comput. Geosci, 20(5), 953-973. doi: 10.1007/s10596-016-9571-6.

Aziz, K. \& Settari, A. (2002). Petrolium Reservoir Simulation. Calgary, Alberta: Blitzprint Ltd.

Barenblat, G. I., Entov, V. M., \& Ryzhik, V. M. (1972). Theory of non-stationary filtration of liquid and gas. MOSCOW: Nedra. 
Barenblatt, G. I., Zheltov, Yu. P., \& Kochina, I. N. (1960). Basic concepts in the theory of seepage of homogeneous fluids in fissurized rocks. Journal of Applied Mathematics and Mechanics, 24(5), 852-864.

Bourgeat, A. (1984). Homogenized behavior of two-phase flows in naturally fractured reservoirs with uniform fractures distribution. Comp. Methods Appl. Mech. Eng, 47(1), 205-216.

Brezzi, F. \& Fortin, M. (1991). Mixed and Hybrid Finite Element Methods. New York: Springer. Verlag. MR1115205

Douglas J. Jr., Arbogast T., Paes-Leme P.J., Hensley J.L., Nunes N.P. (1993). Immiscible displacement in vertically fractured reservoirs. Transp. Porous Media-12(1),73-106. doi: 10.1007/BF00616363.

Efendiev, Y., Lee, S., Li, G., Yao, J., \& Zhang, N. (2015). Hierarchical multiscale modeling for flows in fractured media using generalized multiscale finite element method. Int. J. Geomath, 6(2), 141-162. doi: 10.1007/s13137-015-0075-7.

Ewing, R. (2001). Mathematical modeling and simulation for fluid flow in porous media. Mathematical Modelling, 13(2), 117-127. MR1861667.

Barenblatt, G. I., Entov, V. M., \& Ryzhik, V. M. (1984). The motion of fluids and gases in natural strata. MOSCOW: Nedra.

Glushkova, A. A., \& Papin, A. A. (2019). Stability of two-phase flows in a poroelastic medium. Proceedings of the Seminar on Geometry and Mathematical Modeling-5 (pp.55-59).

Gong, B., Karimi-Fard, M., \& Durlofsky, L. J. (2008). Upscaling discrete fracture characterizations to dual-porosity, dual-permeability models for efficient simulation of flow with strong gravitational effects. SPE J., 13(1), 58-67. doi: 10.2118/102491-PA.

Kalinkin, A. A., Laevsky, Y. M. (2015). Mathematical model of water-oil displacement in fractured porous medium. Sib. Electron. Math. Rep, 12, 743-751.

Kazemi, H., Merrill, L. S. Jr., Porterfield, K. L., \& Zeman, P. R. (1976). Numerical simulation of wateroil flow in naturally fractured reservoirs. Soc. Pet. Eng. J., 16(6), 317-326.

Konovalov, A. N. (1994). Problems of Multiphase Fluid Filtration. New Jersey-London-Hong Kong: World Scientific. Zbl 0837.76002

Kuznetsov,S. V. \& Trofimov, V. A. (1998). Analysis of gas-pressure measurements in coal seams in connection with the manifestation of permeability around holes and mine workings. Journal of Mining Science, 34(2), 113-118.

Kuznetsov, S. V., \& Krigman, R.N. (1978). Natural permeability of coal beds and methods for its determination. Moscow: Nauka.

Kuznetsov, S. V., Trofimov, V. A. (1999). Basic problem of the theory of gas filtration in coal seams. Journal of Mining Science, 35(5), 455-460.

Kuznetsov, S. V., Trofimov, V. A. (2000). Basic provisions and characteristic features of gas filtration in coal seams. Mining Information and Analytical Bulletin, 8, 79-84. 
Kuznetsov, S. V., Trofimov, V. A. (2012). Deformation and destruction of the bottom-hole part of the formation during sudden squeezing. Nonlinear geomechanical-geodynamic processes during the development of mineral deposits at great depths. Novosibirsk.

Kuznetsov, S. V., \& Trofimov, V. A. (2008). Gas dynamics of a coal seam, numerical algorithm, particular and approximate solutions. Mining Information and Analytical Bulletin, 4, 304-324.

Kuznetsov, S. V., \& Trofimov, V. A. (2005), Steady-state gas-and-fluid filtration in a coal seam relative to advancing free surface. Journal of Mining Science, 41(5), 393-398.

Nigmatulin, R. I. (1987). Dynamics of Multiphase Media Part II. Moscow: Nauka.

Trofimov, V. A., \& Filippov, Yu. A. (2015). Peculiarities of deformation of the edge part of a coal seam during "sudden squeezing". Fundamental and applied issues of mining sciences, 2, 284-290.

Trofimov, V. A. (2014). "Out-of-pocket extraction" of the edge of the formation: GIAB.

Vabishchevich, P. N., \& Grigoriev, A. V. (2016). Numerical modeling of a fluid flow in anisotropic fractured porous media. Numerical Analysis and Applications, 9, 45-56.

Vasilyeva, M. V., Vasilyev, V. I., \& Timofeeva, T. S. (2016). Numerical solution of the convective and diffusive transport problems in a heterogeneous porous medium using finite element method. Kazan: Kazan University. pp. 243-261.

Vasilyeva, M. V., Vasilyev, V. I, Grigoriev, A. V., \& Prokopiev, G. A. (2018). Mathematical modeling of the two-phase fluid flow in inhomogeneous fractured porous media using the double porosity model and finite element method. Scientific notes of Kazan University. Series: Physics and Mathematics, 160(1), 165-182.

Vasilyeva, M. V., Vasilyev, V. I., Krasnikov, A. A., \& Nikiforov, D. Ya. (2017). Numerical simulation of single-phase fluid flow in fractured porous media. Kazan: Kazan University. pp. 100-115, 159.

Warren, J. E., \& Root, P. J. (1963). The behavior of naturally fractured reservoirs. Soc. Pet. Eng. J., 3(3), 245-255. doi: 10.2118/426-PA.

Zakharov,V. N., Malinnikova, O. N., Trofimov, V. A., \& Filippov, Yu. A. (2016). The dependence of coal seam permeability on gas content and effective stresses. Physical and technical issues of mining, 2, 16-25.

\section{SEMBLANCE OF THE AUTHOR}

Vitaly A. Trofimov: He is Full Doctor in Technology, Institute of Comprehensive Exploitation of

Mineral Resources Russian Academy of Sciences. His fields of scientific interests include model of two-phase filtration of methane and formation water in the edge of the coal seam. 